\title{
ON THE SQUARE ROOT OF THE CENTRE OF THE HECKE ALGEBRA OF TYPE $A$
}

\author{
ANDREW FRANCIS ${ }^{\curvearrowleft}$ and LENNY JONES
}

(Received 7 December 2004; revised 31 January 2006)

Communicated by J. Du

\begin{abstract}
In this paper we investigate non-central elements of the Iwahori-Hecke algebra of the symmetric group whose squares are central. In particular, we describe a commutative subalgebra generated by certain non-central square roots of central elements, and the generic existence of a rank-three submodule of the Hecke algebra contained in the square root of the centre, but not in the centre. The generators for this commutative subalgebra include the longest word and elements related to trivial and sign characters of the Hecke algebra. We find elegant expressions for the squares of such generators in terms of both the minimal basis of the centre and the elementary symmetric functions of Murphy elements.
\end{abstract}

2000 Mathematics subject classification: primary $20 \mathrm{C} 08$.

\section{Introduction}

In [5], we determined explicitly, for the centre of the Hecke algebra of type $A$, how to express each element of the $\mathbb{Q}\left[q, q^{-1}\right]$-norm basis in [8] as a linear combination of the elements of the $\mathbb{Z}\left[q, q^{-1}\right]$-minimal basis (see [4]). During our research for [5], we were led naturally to the square of the element of the Hecke algebra corresponding to the longest word in the symmetric group. This square, which arises in the definition of certain norms, is well-known to be central, and to have an expression as a product of Murphy elements, which follows from its analogue in the braid group. We derive directly a corresponding expression as a weighted sum of elementary symmetric functions of Murphy elements.

Motivated by recent conversations with John Murray, we have also investigated what other non-central elements of the Hecke algebra might have squares that are

(C) 2007 Australian Mathematical Society $1446-7887 / 07 \$ A 2.00+0.00$ 
central. An analogous study by Murray for the centre of the symmetric group algebra in the modular situation appears in [12]. Results of a general nature arising from our investigation can be found in Section 3, while more detailed specific cases are handled in Section 4. The non-central elements we describe in Section 3 are closely related to well-known central elements of the Hecke algebra, which are important in the study of its representation theory. Together with the longest word, these elements span a submodule contained inside the square root of the centre. As with the element of the Hecke algebra corresponding to the longest word of the symmetric group, the squares of these elements can be expressed as linear combinations of elementary symmetric functions of Murphy operators together with the identity (Theorem 3.8).

\section{Definitions}

Let $S=\left\{s_{1}, \ldots, s_{n-1}\right\}$ be the standard generators of the symmetric group $S_{n}$, meaning that $s_{i}=(i i+1)$ as a permutation. We say an expression $w=s_{i_{1}} \cdots s_{i_{k}} \in S_{n}$, $s_{i_{j}} \in S$, is reduced if $k$ is minimal. In this case, we say the length of $w$, denoted by $l(w)$, is $k$. A partition of $n$ is a composition whose components are weakly decreasing from left to right. If $\lambda$ is a partition of $n$ we write $\lambda \vdash n$. The conjugacy classes in $S_{n}$ are indexed by partitions of $n$.

There are two fairly standard and closely related sets of generators and relations with which to define the Hecke algebra $\mathcal{H}:=\mathcal{H}_{n}$ of $S_{n}$. Let $q$ be an indeterminate and let $R=\mathbb{Z}\left[q^{1 / 2}, q^{-1 / 2}\right]$. Then $\mathcal{H}$ is the associative $R$-algebra generated by the set $\left\{T_{s} \mid s \in S\right\}$ with identity $T_{1}$ and subject to the relations:

$$
\begin{aligned}
T_{s}^{2} & =q T_{1}+(q-1) T_{s}, & & \text { for } s \in S, \\
T_{s_{i}} T_{s_{i+1}} T_{s_{i}} & =T_{s_{i+1}} T_{s_{i}} T_{s_{i+1}}, & & \text { for } 1 \leq i \leq n-2, \\
T_{s_{i}} T_{s_{j}} & =T_{s_{j}} T_{s_{i}} & & \text { for }|i-j| \geq 2 .
\end{aligned}
$$

The algebra $\mathcal{H}$ is a free $R$-module with basis $\left\{T_{w}:=T_{s_{i_{1}}} \ldots T_{s_{i_{r}}} \mid w \in S_{n}\right\}$ where $w=s_{i_{1}} \cdots s_{i_{r}}$ is a reduced expression for $w$ in $S_{n}$. We present most of the results in this paper in terms of this set of generators and relations, and with respect to this basis of $\mathcal{H}$ as a module. However, some results (Section 2) are more elegantly expressed in terms of a normalised generating set $\left\{\tilde{T}_{s} \mid s \in S\right\}$, defined by setting $\tilde{T}_{s}:=q^{-1 / 2} T_{s}$. If we set $\xi=q^{1 / 2}-q^{-1 / 2}$ then the new generators satisfy the braid relations (1.2) and (1.3), but the order relation (1.1) becomes

$$
\tilde{T}_{s}^{2}=\tilde{T}_{1}+\xi \tilde{T}_{s}
$$

We indicate corresponding elements and substructures of this normalised version of the Hecke algebra by placing a tilde above them. For example, we denote the normalised 
Hecke algebra itself as $\tilde{\mathcal{H}}$. Then $\tilde{\mathcal{H}}$ is a free $\mathbb{Z}[\xi]$-module with basis $\left\{\tilde{T}_{w}:=\tilde{T}_{s_{i_{1}}} \ldots \tilde{T}_{s_{i_{r}}}\right\}$ where $w=s_{i_{1}} \cdots s_{i_{r}}$ is a reduced expression for $w$ in $S_{n}$.

The symmetric group $S_{n}$ has a symmetry best described as the graph automorphism induced by reflecting the Dynkin diagram about its midpoint. If $\rho_{n}: S_{n} \rightarrow S_{n}$ is the group automorphism defined by this symmetry then $\rho_{n}\left(s_{i}\right)=s_{n-i}$ for $1 \leq i \leq n-1$. This automorphism is naturally extended to an algebra automorphism of $\mathcal{H}$, also denoted $\rho_{n}$.

The centre $Z$ of $\mathcal{H}$ is defined to be the set of elements $c \in \mathcal{H}$ such that $c h=h c$ for all $h \in \mathcal{H}$. Throughout this paper we make frequent use of the 'minimal basis' for $Z$ given in $([4,7])$. This minimal basis $\left\{\Gamma_{\lambda} \mid \lambda \vdash n\right\}$ is an $R$-basis for $Z$ and can be characterized by the following two properties:

- $\left.\Gamma_{\lambda}\right|_{q=1}=\sum_{w \in C_{\lambda}} T_{w}$, where $C_{\lambda}$ is the conjugacy class of cycle type $\lambda$ in $S_{n}$, and

- $\Gamma_{\lambda}-\sum_{w \in C_{\lambda}} T_{w}$ contains no minimal length elements from any conjugacy class.

The corresponding class elements in $\tilde{\mathcal{H}}$ are $\left\{\tilde{\Gamma}_{\lambda} \mid \lambda \vdash n\right\}$ with the characterizing properties that $\left.\tilde{\Gamma}_{\lambda}\right|_{\xi=0}=\sum_{w \in C_{\lambda}} \tilde{T}_{w}$, and that $\tilde{\Gamma}_{\lambda}-\sum_{w \in C_{\lambda}} \tilde{T}_{w}$ contains no minimal length elements from any conjugacy class. We have $\tilde{\Gamma}_{\lambda}=q^{-l_{\lambda} / 2} \Gamma_{\lambda}$, where $l_{\lambda}$ is the length of the shortest elements in $C_{\lambda}$.

Write $w_{n}$ for the longest word in $S_{n}$. Define $\ell:=l\left(w_{n}\right)=\sum_{i=1}^{n-1} i$, the length of the longest word.

Define the Poincaré polynomial to be $p(q):=\sum_{w \in S_{n}} q^{l(w)}$.

\section{The square of the element in $\tilde{\mathcal{H}}$ corresponding to $w_{n}$}

In this section we use the normalized version of the Hecke algebra $\tilde{\mathcal{H}}$ to give an explicit and surprisingly simple expression for $\tilde{T}_{w_{n}}^{2}$.

We begin with some basic facts about Murphy elements.

DEFINITION 2.1. The Murphy operators or Murphy elements $L_{n, i}$ of $\mathcal{H}$ are defined by setting $L_{n, 1}=0$ and

$$
\begin{aligned}
L_{n, i} & =T_{(i-1 i)}+q^{-1} T_{(i-2 i)}+\cdots+q^{-(i-2)} T_{(1 i)} \\
& =T_{s_{i-1}}+q^{-1} T_{s_{i-2} s_{i-1} s_{i-2}}+\cdots+q^{-(i-2)} T_{s_{1} \cdots s_{i-1} \cdots s_{1}}
\end{aligned}
$$

for $i=2,3, \ldots, n$.

By a symmetric function in a set of commuting variables, we mean a polynomial in those variables that is unchanged by any permutation of the variables.

THEOREM 2.2 (Dipper-James [2] or Murphy [9]). The Murphy elements commute. The symmetric functions in the Murphy elements are in $Z$. 
The following was shown by Dipper and James [2] when the Hecke algebra is defined over a suitable field (such as $\mathbb{Q}\left(q^{1 / 2}\right)$ ), and by Francis and Graham [6] over $R$.

THEOREM 2.3 (Dipper-James [2], Francis-Graham [6]). The set of symmetric functions in the Murphy elements in $\mathcal{H}$ is precisely $Z$.

The Murphy elements can be similarly defined in $\tilde{\mathcal{H}}$ by setting $\tilde{L}_{n, 1}:=0$ and $\tilde{L}_{n, i}:=\tilde{T}_{(i-1) i)}+\tilde{T}_{(i-2 i)}+\cdots+\tilde{T}_{(1 i)}$. Of course, the statement of Theorem 2.2 readily translates to the normalised context.

The $i$-th elementary symmetric function in $n$ commuting variables is the sum of all monomials of length $i$ in the variables whose exponents are at most 1. For example, the second elementary symmetric function in the four variables $x_{1}, x_{2}$, $x_{3}, x_{4}$ is $x_{1} x_{2}+x_{1} x_{3}+x_{1} x_{4}+x_{2} x_{3}+x_{2} x_{4}+x_{3} x_{4}$. Define $\tilde{e}_{n, 0}:=\tilde{T}_{1}$, and for $i=1, \ldots, n-1$, let $\tilde{e}_{n, i}$ denote the $i$-th elementary symmetric function in the Murphy elements $\tilde{L}_{n, 1}, \ldots, \tilde{L}_{n, n}$.

PROPOSITION 2.4 ([6]). For $i=0, \ldots, n-1, \tilde{e}_{n, i}=\sum_{l_{\lambda}=i} \tilde{\Gamma}_{\lambda}$. Moreover, we have the corresponding $e_{n, i}=\sum_{l_{\lambda}=i} \Gamma_{\lambda}$.

COROLlARY 2.5. The set $\left\{\sum_{l_{\lambda}=i} \Gamma_{\lambda} \mid 1 \leq i \leq n-1\right\}$ generates $Z$ over $R$.

Set $\tilde{M}_{n, i}:=\rho_{n}\left(\tilde{L}_{n, i}\right)=\tilde{T}_{(n n-i+1)}+\cdots+\tilde{T}_{(n-i+2 n-i+1)}$, for $1 \leq i \leq n$. We call the $\tilde{M}_{n, i}$ the dual Murphy elements. These elements also appear in [13, page 26].

For example, in $S_{4}$

$$
\begin{aligned}
& \tilde{M}_{4,1}=0, \quad \tilde{M}_{4,2}=\tilde{T}_{(43)}=\tilde{T}_{s_{3}}, \\
& \tilde{M}_{4,3}=\tilde{T}_{(42)}+\tilde{T}_{(32)}=\tilde{T}_{s_{2} s_{3} s_{2}}+\tilde{T}_{s_{2}}, \\
& \tilde{M}_{4,4}=\tilde{T}_{(41)}+\tilde{T}_{(31)}+\tilde{T}_{(21)}=\tilde{T}_{s_{1} s_{2} s_{3} s_{2} s_{1}}+\tilde{T}_{s_{1} s_{2} s_{1}}+\tilde{T}_{s_{1}} .
\end{aligned}
$$

These elements are 'dual' because if you add 'down the columns' instead of along the rows you get the standard Murphy elements. That is, adding the first terms of each element one has $\tilde{L}_{4,4}$, adding the second terms gives $\tilde{L}_{4,3}$, and adding the third terms gives $\tilde{L}_{4,2}$.

If you like, the $i$-th Murphy element 'hangs down' from $i$ to 1 , while the dual version 'grows up' from $n-i+1$ to $n$.

LEMMA 2.6.

(1) $\sum_{i=1}^{n} \tilde{M}_{n, i}=\sum_{i=1}^{n} \tilde{L}_{n, i}$.

(2) $\tilde{M}_{i, i}=\tilde{M}_{i-1, i-1}+\tilde{T}_{(1 i)}$ for $i \geq 2$.

(3) $\tilde{T}_{s_{1} \ldots s_{n}} \tilde{T}_{s_{n} \ldots s_{1}}=\tilde{T}_{1}+\xi \tilde{M}_{n+1 . n+1}$. 
(4) For $i=0,1, \ldots, n-2, \tilde{L}_{n+1, n+1} \tilde{e}_{n, i}+\tilde{e}_{n, i+1}=\tilde{e}_{n+1, i+1}$. For $i=n-1$ we have $\tilde{L}_{n+1, n+1} \tilde{e}_{n, n-1}=\tilde{e}_{n+1, n}$.

(5) $\rho_{n}\left(\tilde{e}_{n, i}\right)=\tilde{e}_{n, i}$ for $i=0,1, \ldots, n-1$.

PROOF. (1) The sums on each side are equal to $\sum_{1 \leq i<j \leq n} \tilde{T}_{(i j)}$.

(2) This follows by definition of $\tilde{M}_{i, j}$.

(3) This involves a straightforward induction on $n$ using (2).

(4) These are elementary properties of symmetric functions in commuting variables.

(5) This also follows since $\tilde{e}_{n, i}$ is symmetric not only in the Murphy elements but also in the $s_{i}$ under $\rho_{n}$.

The following result (equation (2.1)) is stated in [14, Section 3] and [13, Section 6] using an alternative definition of Murphy elements in $\tilde{\mathcal{H}}$, defined by setting $\mathcal{L}_{n, 1}=1$ and $\mathcal{L}_{n, i+1}=\tilde{T}_{s_{i}} \mathcal{L}_{n, i} \tilde{T}_{s_{i}}$. It can be proved by induction on $n$, but is also a direct consequence of looking at the Hecke algebra as a quotient of the Braid group algebra. In the braid group, the square of the longest word is the 'full twist', and the Murphy element $\mathcal{L}_{n, i}$ is the braid whose $i$-th string goes behind strings $i-1, \ldots, 1$ and returns in front of strings $1, \ldots, i-1$ to the $i$-th position. All other strings remain as in the identity braid. It immediately follows that

$$
\tilde{T}_{w_{n}}^{2}=\prod_{i=1}^{n} \mathcal{L}_{n, i} .
$$

In Theorem 2.7 we present an alternative expression for $\tilde{T}_{w_{n}}^{2}$, which is a direct consequence of (2.1) together with the relation $\mathcal{L}_{n, i}=\xi \tilde{L}_{n, i}+\tilde{T}_{1}$ (the proof of this relation is elementary). We provide a direct independent proof here.

THEOREM 2.7. $\tilde{T}_{w_{n}}^{2}=\sum_{i=0}^{n-1} \xi^{i} \tilde{e}_{n, i}$.

PROOF. The proof is by induction on $n$. If $n=2$, then

$$
\tilde{T}_{w_{2}}^{2}=\tilde{T}_{s_{1}}^{2}=\tilde{T}_{1}+\xi \tilde{T}_{s_{1}}=\tilde{e}_{2,0}+\xi \tilde{e}_{2,1}
$$

since $\tilde{e}_{2,1}=\tilde{T}_{s_{1}}$. Suppose the statement is true for $n$. Now $w_{n+1}=s_{1} \cdots s_{n} w_{n}=$ $w_{n} s_{n} \cdots s_{1}$. Also, it is clear that $w_{n} s_{n} \cdots s_{1}=s_{n} \cdots s_{1} \rho_{n+1}\left(w_{n}\right)$. So, using the fact that $\rho_{n+1}$ is an algebra automorphism,

$$
\begin{aligned}
\tilde{T}_{w_{n+1}}^{2} & =\tilde{T}_{s_{1} \ldots s_{n}} \tilde{T}_{w_{n}}^{2} \tilde{T}_{s_{n} \ldots s_{1}}=\tilde{T}_{s_{1} \ldots s_{n}} \tilde{T}_{s_{n} \cdots s_{1}} \rho_{n+1}\left(\tilde{T}_{w_{n}}^{2}\right) \\
& =\rho_{n+1}\left(\mathcal{L}_{n+1, n+1}\right) \rho_{n+1}\left(\sum_{i=0}^{n-1} \xi^{i} \tilde{e}_{n, i}\right) \quad \text { by induction }
\end{aligned}
$$




$$
\begin{aligned}
= & \rho_{n+i}\left(\left(\tilde{T}_{1}+\xi \tilde{L}_{n+1, n+1}\right)\left(\sum_{i=0}^{n-1} \xi^{i} \tilde{e}_{n, i}\right)\right) \\
= & \rho_{n+1}\left(\tilde{T}_{1}+\xi\left(\tilde{L}_{n+1, n+1} \tilde{e}_{n, 0}+\tilde{e}_{n, 1}\right)+\xi^{2}\left(\tilde{L}_{n+1, n+1} \tilde{e}_{n, 1}+\tilde{e}_{n, 2}\right)\right. \\
& \left.+\cdots+\xi^{n-1}\left(\tilde{L}_{n+1, n+1} \tilde{e}_{n, n-2}+\tilde{e}_{n, n-1}\right)+\xi^{n} \tilde{L}_{n+1, n+1} \tilde{e}_{n, n-1}\right) \\
= & \rho_{n+1}\left(\tilde{T}_{1}+\xi \tilde{e}_{n+1,1}+\xi^{2} \tilde{e}_{n+1,2}+\cdots+\xi^{n} \tilde{e}_{n+1, n}\right) \\
= & \tilde{T}_{1}+\xi \tilde{e}_{n+1,1}+\xi^{2} \tilde{e}_{n+1,2}+\cdots+\xi^{n} \tilde{e}_{n+1, n},
\end{aligned}
$$

as desired (the last equality is due to Lemma $2.6(5)$, and the second to last due to Lemma $2.6(4))$.

COROLLARY 2.8. In terms of class elements, $\tilde{T}_{w_{n}}^{2}=\sum_{\lambda \vdash n} \xi^{l_{\lambda}} \tilde{\Gamma}_{\lambda}$.

PROOF. This is an immediate consequence of Theorem 2.7 and Proposition 2.4.

Lastly, we rewrite Corollary 2.8 over $R$.

Recall the transformations $\tilde{T}_{s}=q^{-1 / 2} T_{s}$ and $\xi=q^{1 / 2}-q^{-1 / 2}$. So $\tilde{T}_{w_{n}}^{2}=$ $\left(q^{-\ell / 2} T_{w_{n}}\right)^{2}=q^{-\ell} T_{w_{n}}^{2}$. We also have $\tilde{\Gamma}_{\lambda}=q^{-l_{\lambda} / 2} \Gamma_{\lambda}$, and so

$$
\xi^{l_{\lambda}} \tilde{\Gamma}_{\lambda}=q^{-l_{\lambda} / 2}\left(q^{1 / 2}-q^{-1 / 2}\right)^{l_{\lambda}} \Gamma_{\lambda}=q^{-l_{\lambda}}(q-1)^{l_{\lambda}} \Gamma_{\lambda}
$$

Recall from Proposition 2.4 that $e_{n, i}=\sum_{l_{\lambda}=i} \Gamma_{\lambda}$. Therefore, the statement of Corollary 2.8 can be written as follows.

COROLLARY 2.9. $T_{w_{n}}^{2}=q^{\ell} \sum_{\lambda \vdash n}\left(1-q^{-1}\right)^{l_{\lambda}} \Gamma_{\lambda}$.

\section{The square root of the centre of $\mathcal{H}$}

When $n=2$ the algebra $\mathcal{H}_{n}$ is commutative, so we restrict our attention in this section to $n \geq 3$.

DEFINITION 3.1. The square root of $Z$, denoted $\sqrt{Z}$, is defined to be

$$
\sqrt{Z}:=\left\{h \in \mathcal{H} \mid h^{2} \in Z\right\}
$$

Clearly $Z \subseteq \sqrt{Z}$. Also, $Z \neq \sqrt{Z}$ since $T_{w_{n}} \in \sqrt{Z} \backslash Z$. The fact that $T_{w_{n}} \in \sqrt{Z} \backslash Z$ is well-known and follows from the centrality of the square of the corresponding longest element in the braid group (of which $\mathcal{H}$ is a quotient), where that square is the full twist. In this section we define two additional elements, which we show are in $\sqrt{Z}$, 
and together with $T_{w_{n}}$, generate a commutative subalgebra of $\mathcal{H}$. In Theorem 3.8 we give the forms of the squares of these elements.

We say an element $v \in \mathcal{H}$ is a right eigenvector for multiplication by $h$ if $h v=k v$ for some $k \in R$. Left eigenvectors are defined similarly.

Recall the following well-known elements of $Z$ :

$$
x:=\sum_{w \in S_{n}} T_{w}, \quad y:=\sum_{w \in S_{n}}(-q)^{\ell-l(w)} T_{w} .
$$

These elements were defined in [1, Section 3] and used to study the permutation modules of $\mathcal{H}$. They are widely used in the representation theory of $\mathcal{H}$ (see, for example, [2, 3, 10], and [11]). The modules $x \mathcal{H}$ and $y \mathcal{H}$ are called the trivial and alternating $\mathcal{H}$-modules, respectively. Analogous elements can be defined for parabolic subalgebras of $\mathcal{H}$. Both $x$ and $y$ are eigenvectors for the action of the generators of $\mathcal{H}$ (Lemma 3.2 (1)), a property that they share with some other elements of $\sqrt{Z}$ (see Section 4).

The following result is well-known.

LEMMA 3.2 .

(1) For any $s \in S, T_{s} x=x T_{s}=q x$ and $T_{s} y=y T_{s}=-y$. Moreover, $x$ and $y$ are central.

(2) $x y=0$.

(3) $x=\sum_{\lambda \vdash n} \Gamma_{\lambda}$ and $y=\sum_{\lambda \vdash n}(-q)^{\ell-l_{\lambda}} \Gamma_{\lambda}$.

(4) $x^{2}=p(q) x$ and $y^{2}=(-1)^{\ell} p(q) y$.

We now define the elements $\bar{x}$ and $\bar{y}$, and investigate their properties in the remainder of this paper:

$$
\bar{x}:=x-T_{w_{n}}, \quad \bar{y}:=y-T_{w_{n}} .
$$

LEMMA 3.3. The following hold:

(1) $\bar{x} T_{w_{n}}=T_{w_{n}} \bar{x}$,

(2) $\bar{y} T_{w_{n}}=T_{w_{n}} \bar{y}$,

(3) $\bar{x} T_{w_{n}}, \bar{y} T_{w_{n}}, \bar{x} \bar{y} \in Z$,

(4) $\bar{x} \bar{y}=\bar{y} \bar{x}$.

ProOF. The centrality of $x$ and $y$ from Lemma 3.2 (1) implies (1) and (2). Now $w_{n}$ acts on the set $S$ of generators of $S_{n}$ via the graph automorphism $\rho_{n}$ defined in Section 1, and this action extends to the Hecke algebra level as follows (remembering that $l\left(s w_{n}\right)=l\left(w_{n} s\right)=l\left(w_{n}\right)-1$ for any $\left.s \in S\right)$ :

$$
T_{w_{n}} T_{s}=T_{\rho_{n}(s)} T_{\rho_{n}(s) w_{n}} T_{s}=T_{\rho_{n}(s)} T_{w_{n} s} T_{s}=T_{\rho_{n}(s)} T_{w_{n}} .
$$


Since $T_{s} x=x T_{s^{\prime}}$ for any $s, s^{\prime} \in S$, we have in particular $T_{s} x=x T_{\rho_{n}(s)}$. Hence, for any $s \in S$ (using the fact that $\rho_{n}$ is an involution), we have

$$
\begin{aligned}
\bar{x} T_{w_{n}} T_{s} & =\bar{x} T_{\rho_{n}(s)} T_{w_{n}}=\left(x-T_{w_{n}}\right) T_{\rho_{n}(s)} T_{w_{n}}=\left(x T_{\rho_{n}(s)}-T_{w_{n}} T_{\rho_{n}(s)}\right) T_{w_{n}} \\
& =\left(T_{s} x-T_{s} T_{w_{n}}\right) T_{w_{n}}=T_{s}\left(x-T_{w_{n}}\right) T_{w_{n}}=T_{s} \bar{x} T_{w_{n}} .
\end{aligned}
$$

Therefore, $\bar{x} T_{w_{n}} \in Z$. Similar arguments show that $\bar{y} T_{w_{n}}$ and $\bar{x} \bar{y}$ are central, proving (3). Finally, the centrality of $x$ implies $\bar{y} x=x \bar{y}$, which together with (2) implies (4), and the proof is complete.

PROPOSITION 3.4. The elements $\bar{x}, \bar{y}, T_{w_{n}}$ are in $\sqrt{Z} \backslash Z$.

Proof. As already mentioned, it is well-known that $T_{w_{n}} \in \sqrt{Z} \backslash Z$. By Lemma 3.2 (1), we have that $x, y \in Z$, from which we deduce that $\bar{x}, \bar{y} \notin Z$. Since $x^{2}, y^{2}, T_{w_{n}}^{2} \in Z$, and since $T_{w_{n}}$ commutes with $\bar{x}$ and $\bar{y}$ (Lemma 3.3), it follows that $\bar{x}^{2}, \bar{y}^{2} \in Z$.

REMARK. While $\bar{x}+\bar{y} \notin Z$, some linear combinations of $\bar{x}$ and $\bar{y}$ are in $Z$ (for instance $\bar{x}-\bar{y}$ ), and all linear combinations of $\bar{x}$ and $\bar{y}$ are in $\sqrt{Z}$ since $\bar{x}^{2}, \bar{y}^{2}, \bar{x} \bar{y} \in Z$.

The following is an immediate consequence of Lemma 3.3 and Proposition 3.4.

COROllaRY 3.5. The $R$-submodule of $\mathcal{H}$ spanned by $\left\{\bar{x}, \bar{y}, T_{w_{n}}\right\}$ is contained in $\sqrt{Z} \backslash Z$.

PROPOSITION 3.6. The elements $\bar{x}$ and $\bar{y}$ are not zero divisors.

PROOF. Suppose that $\bar{x} h=\left(x-T_{w_{n}}\right) h=0$ for some $h \in \mathcal{H}$. Multiplying on the left by $x$ gives $\left(x^{2}-x T_{w_{n}}\right) h=0$, and therefore, using Lemma 3.2 (1) and (4), we have $\left(p(q)-q^{l\left(w_{n}\right)}\right) x h=0$. This gives $x h=0$ since $\mathcal{H}$ is a free $R$-module, and subtracting $x h=0$ from $\left(x-T_{w_{n}}\right) h=0$ we obtain $T_{w_{n}} h=0$. Thus $h=0$, since $T_{w}$ is invertible for all $w \in S_{n}$. The proof for $\bar{y}$ is similar.

PROPOSITION 3.7. Let $\mathcal{X}$ be the $R$-subalgebra of $\mathcal{H}$ generated by $\left\{\bar{x}, \bar{y}, T_{w_{n}}\right\}$. Then $\mathcal{X}$ is commutative.

PROOF. This is immediate from Lemma 3.3.

REMARK. Since $\bar{x} \bar{y} T_{w_{n}} \notin Z$, we have that $\bar{x}+\bar{y} T_{w_{n}} \notin \sqrt{Z}$. Hence, $\mathcal{X} \nsubseteq \sqrt{Z}$. This also shows that $\sqrt{Z}$ itself is not a subalgebra of $\mathcal{H}$. However, the set $\left\{\bar{x}^{a} \bar{y}^{b} T_{w_{n}}^{c}\right\}$ $a, b, c \in \mathbb{N}\} \subseteq \sqrt{Z}$, and, in fact, all elements of $\mathcal{X}$ with even exponent sum are central. 
Unfortunately, $\sqrt{Z}$ (which includes $Z$ ) is not even an $R$-module. Although we have shown that all $R$-linear combinations of the elements $\bar{x}, \bar{y}$ and $T_{w_{n}}$ are contained in $\sqrt{Z}$ (Corollary 3.5), it is not true in general that other elements of $\sqrt{Z}$ have this property (see Section 4 ), and certainly unlikely that the sum of a non-central element in $\sqrt{Z}$ and an element of $Z$ is in $\sqrt{Z}$.

REMARK. There are no subalgebras of $\mathcal{H}$, which are contained in $\sqrt{Z}$, that contain elements in $\sqrt{Z} \backslash Z$. This is because, for instance, if $h \in \sqrt{Z} \backslash Z$, then $h+h^{2} \notin \sqrt{Z}$.

THEOREM 3.8. The squares of $\bar{x}$ and $\bar{y}$ can be expressed as linear combinations of elementary symmetric functions of Murphy elements together with the identity. Specifically,

$$
\begin{aligned}
\vec{x}^{2} & =\sum_{\lambda \vdash n}\left(p(q)-2 q^{\ell}+q^{\ell-l_{\lambda}}(q-1)^{l_{\lambda}}\right) \Gamma_{\lambda} \\
& =\sum_{i=0}^{n-1}\left(p(q)-2 q^{\ell}+q^{\ell-i}(q-1)^{i}\right) e_{n, i},
\end{aligned}
$$

and

$$
\begin{aligned}
\bar{y}^{2} & =\sum_{\lambda \vdash n}(-1)^{l_{\lambda}} q^{\ell-l_{\lambda}}\left(p(q)-2+(1-q)^{l_{\lambda}}\right) \Gamma_{\lambda} \\
& =\sum_{i=0}^{n-1}(-1)^{i} q^{\ell-i}\left(p(q)-2+(1-q)^{i}\right) e_{n, i} .
\end{aligned}
$$

PROOF. Immediately from the definition of $\bar{x}$ and $\bar{y}$ we have that

$$
\bar{x}^{2}=x^{2}-2 x T_{w_{n}}+T_{w_{n}}^{2}, \quad \text { and } \quad \bar{y}^{2}=y^{2}-2 y T_{w_{n}}+T_{w_{n}}^{2} .
$$

The forms for $x^{2}, y^{2}$ and $T_{w_{n}}^{2}$ from Lemma 3.2 (3) and (4) and Corollary 2.9, together with Lemma 3.2 (1), then give that

$$
\bar{x}^{2}=p(q) x-2 q^{\ell} x+T_{w_{n}}^{2}=\left(p(q)-2 q^{\ell}\right) \sum_{\lambda \vdash n} \Gamma_{\lambda}+q^{\ell} \sum_{\lambda \vdash n}\left(1-q^{-1}\right)^{l_{\lambda}} \Gamma_{\lambda} .
$$

The expression for $\bar{x}^{2}$ in terms of $\left\{\Gamma_{\lambda} \mid \lambda \vdash n\right\}$ follows. The corresponding expression for $\bar{y}^{2}$ is similarly derived. The expressions for both $\bar{x}^{2}$ and $\bar{y}^{2}$ in terms of $\left\{e_{n, i} \mid 0 \leq i \leq n-1\right\}$ follow immediately from Proposition 2.4 .

\section{Examples: $\sqrt{Z}$ for $\mathcal{H}_{3}$ and $\mathcal{H}_{4}$}

We have seen in Section 3 that the commutative subalgebra $\mathcal{X}$ of $\mathcal{H}$ generated by $\left\{\bar{x}, \bar{y}, T_{w_{n}}\right\}$ is not contained in $\sqrt{Z}$. We see in the following examples that it is also the case that $\sqrt{Z}$ is not contained in $\mathcal{X}$. 
4.1. $\mathcal{H}_{3}$. When $n=3$ it is easy to check that, while the elements $T_{s_{1}}-T_{s_{2}}$ and $T_{s_{1} s_{2}}-T_{s_{2} s_{1}}$ are in $\sqrt{Z}$, their span is not in $\sqrt{Z}$ - unlike $\bar{x}_{3}, \bar{y}_{3}$ and $T_{w_{3}}=T_{s_{1} s_{2} s_{1}}$. However, they do have the interesting property that they are eigenvectors for the multiplicative action of $Z$. This parallels a similar fact for $x$ and $y$ (Lemma 3.2 (1)). Recall from Section 1 that $\left\{\Gamma_{(1,1,1)}=T_{1}, \Gamma_{(2,1)}=T_{s_{1}}+T_{s_{2}}+q^{-1} T_{s_{1} s_{2} s_{1}}, \Gamma_{(3)}=T_{s_{1} s_{2}}+\right.$ $\left.T_{s_{2} s_{1}}+q^{-1}(q-1) T_{s_{1} s_{2} s_{1}}\right\}$ is an $R$-basis for $Z$. Then we have

$$
\begin{aligned}
\Gamma_{(2,1)}\left(T_{1}-T_{2}\right) & =(q-1)\left(T_{1}-T_{2}\right), & \Gamma_{(2,1)}\left(T_{12}-T_{21}\right) & =(q-1)\left(T_{12}-T_{21}\right), \\
\Gamma_{(3)}\left(T_{1}-T_{2}\right) & =-q\left(T_{1}-T_{2}\right), & \Gamma_{(3)}\left(T_{12}-T_{21}\right) & =-q\left(T_{12}-T_{21}\right) .
\end{aligned}
$$

PROPOSITION 4.1. When $n=3$, every element in $\sqrt{Z} \backslash Z$ can be written as a $\mathbb{Q}(q)$-linear combination of the following elements:

$$
\begin{aligned}
\bar{x}_{3} & :=T_{1}+T_{s_{1}}+T_{s_{2}}+T_{s_{1} s_{2}}+T_{s_{2} s_{1}}, \\
\bar{y}_{3} & :=T_{1}-q^{-1}\left(T_{s_{1}}+T_{s_{2}}\right)+q^{-2}\left(T_{s_{1} s_{2}}+T_{s_{2} s_{1}}\right), \\
T_{w_{3}} & :=T_{s_{1} s_{2} s_{1}}, \quad R_{4}:=T_{s_{1}}-T_{s_{2}}, \quad R_{5}:=T_{s_{1} s_{2}}-T_{s_{2} s_{1}} .
\end{aligned}
$$

PROOF. Solving the system of equations created by evaluating

$$
\begin{aligned}
\left(a_{1} T_{1}\right. & \left.+a_{2} T_{s_{1}}+a_{3} T_{s_{2}}+a_{4} T_{s_{1} s_{2}}+a_{5} T_{s_{2} s_{1}}+a_{6} T_{s_{1} s_{2} s_{1}}\right)^{2} T_{s} \\
& =T_{s}\left(a_{1} T_{1}+a_{2} T_{s_{1}}+a_{3} T_{s_{2}}+a_{4} T_{s_{1} s_{2}}+a_{5} T_{s_{2} s_{1}}+a_{6} T_{s_{1} s_{2} s_{1}}\right)^{2}
\end{aligned}
$$

for each $s \in S=\left\{s_{1}, s_{2}\right\}$ yields the following two solutions:

$$
\left\{a_{2}=a_{3}, a_{4}=a_{5}, a_{6}=q^{-1} a_{3}+q^{-1}(q-1) a_{5}\right\}
$$

and

$$
\left\{a_{1}=-\frac{q-1}{2}\left(a_{2}+a_{3}\right)+\frac{q}{2}\left(a_{4}+a_{5}\right)\right\} .
$$

The first solution, (4.1), characterizes the elements of $Z$ : the relations given are exactly those given by [4, Lemma 3.1]. Thus the elements of $\sqrt{Z} \backslash Z$ must satisfy equations (4.2). The elements $\left\{\bar{x}_{3}, \bar{y}_{3}, T_{w_{3}}, R_{4}, R_{5}\right\}$ all satisfy these equations, and are easily seen to be linearly independent. Thus they span a five-dimensional subspace of the solution space, which itself has five dimensions (there are five free parameters). Hence they span the solution space of (4.2).

COROLLARY 4.2. For any subset $W$ of $\mathcal{H}$, let $\langle W\rangle$ denote the $R$-span of $W$. Then $\left\langle\left\{\bar{x}_{3}, \bar{y}_{3}, T_{w_{3}}, R_{4}, R_{5}\right\}\right\rangle \cap Z_{3}=\left\langle\left\{x_{3}, y_{3}\right\}\right\rangle$.

PROOF. This is immediate from common solutions to (4.1) and (4.2). 
The squares of these five square roots of central elements (using Theorems 3.8 and 2.7) are:

$$
\begin{aligned}
\bar{x}_{3}^{2} & =\left(2 q^{2}+2 q+1\right) \Gamma_{(1,1,1)}+(q+1)^{2} \Gamma_{(2,1)}+(3 q+1) \Gamma_{(3)}, \\
\bar{y}_{3}^{2} & =q^{4}\left(q^{2}+2 q+2\right) \Gamma_{(1,1,1)}-q^{3}(q+1)^{2} \Gamma_{(2,1)}+q^{3}(q+3) \Gamma_{(3)}, \\
T_{w_{3}}^{2} & =\Gamma_{(1,1,1)}+\left(1-q^{-1}\right) \Gamma_{(2,1)}+\left(1-q^{-1}\right)^{2} \Gamma_{(3)}, \\
R_{4}^{2} & =2 q \Gamma_{(1,1,1)}+(q-1) \Gamma_{(2,1)}-\Gamma_{(3)}, \\
R_{5}^{2} & =-2 q^{2} \Gamma_{(1,1,1)}-q(q-1) \Gamma_{(2,1)}+q \Gamma_{(3)} .
\end{aligned}
$$

Interestingly, we have the relation $R_{5}^{2}=-q R_{4}^{2}$. It follows that the central element given by $R_{4}^{2}$ has at least four distinct square roots over $\mathbb{C}\left(q^{1 / 2}\right)$.

4.2. $\mathcal{H}_{4}$. As with $\mathcal{H}_{3}$, the algebra $\mathcal{X}$ generated by $\left\{\bar{x}_{4}, \bar{y}_{4}, T_{w_{4}}\right\}$ does not contain all elements of $\sqrt{Z} \backslash Z$. Motivated by the relationships among the elements $\bar{x}_{4}, \bar{y}_{4}$ and the eigenvectors of $Z$ when $n=4$, as well as the experience in $\mathcal{H}_{3}$, we have identified several more elements of $\sqrt{Z}$ by looking for eigenvectors of the multiplicative action of class elements. The following are additional examples of square roots, which are clearly non-central since the coefficients of the shortest elements of conjugacy classes are not equal

$$
\begin{aligned}
R_{4}:= & q\left(T_{s_{1} s_{2}}-T_{s_{2} s_{1}}+T_{s_{3} s_{2}}-T_{s_{2} s_{3}}\right)+(q-1)\left(T_{s_{1} s_{3} s_{2}}-T_{s_{2} s_{1} s_{3}}\right) \\
& +T_{s_{1} s_{2} s_{1} s_{3}}-T_{s_{1} s_{2} s_{3} s_{2}}+T_{s_{2} s_{3} s_{2} s_{1}}-T_{s_{1} s_{3} s_{2} s_{1}}, \\
R_{5}:= & q^{2}\left(T_{s_{1}}+T_{s_{3}}\right)+q(q-1)\left(T_{s_{2} s_{1}}+T_{s_{2} s_{3}}+T_{s_{1} s_{3}}\right)+(q-1)^{2} T_{s_{2} s_{1} s_{3}} \\
& -q\left(T_{s_{1} s_{2} s_{1}}+T_{s_{2} s_{3} s_{2}}+T_{s_{1} s_{2} s_{3}}+T_{s_{3} s_{2} s_{1}}\right) \\
& -(q-1)\left(T_{s_{1} s_{2} s_{1} s_{3}}+T_{s_{2} s_{3} s_{2} s_{1}}+T_{s_{2} s_{1} s_{3} s_{2}}\right)+T_{s_{1} s_{2} s_{1} s_{3} s_{2}}+T_{s_{2} s_{1} s_{3} s_{2} s_{1}}, \\
R_{6}:= & q^{2} T_{s_{2}}+q(q-1)\left(T_{s_{1} s_{2}}+T_{s_{3} s_{2}}\right)-q\left(T_{s_{1} s_{2} s_{1}}+T_{s_{s_{2} s_{3} s_{2}}}+T_{s_{1} s_{2} s_{3}}+T_{s_{3} s_{2} s_{1}}-T_{s_{2} s_{1} s_{3}}\right) \\
& +\left(q^{2}-q+1\right) T_{s_{1} s_{3} s_{2}}-(q-1)\left(T_{s_{1} s_{2} s_{3} s_{2}}+T_{s_{1} s_{3} s_{2} s_{1}}\right)+T_{s_{1} s_{2} s_{3} s_{2} s_{1}} .
\end{aligned}
$$

The set $\left\{\bar{x}_{4}, \bar{y}_{4}, T_{w_{4}}, R_{4}, R_{5}, R_{6}\right\}$ is linearly independent. While the elements $\left\{\bar{x}_{4}, \bar{y}_{4}, T_{w_{4}}\right\}$ commute with each other and with elements of $\left\{R_{4}, R_{5}, R_{6}\right\}$, the elements of $\left\{R_{4}, R_{5}, R_{6}\right\}$ do not commute among themselves.

Unlike the $\mathcal{H}_{3}$ case, we cannot use a dimension argument here to prove that we have all square roots. In fact, as the dimension of the algebra here is 24 , it is rather unlikely that this set spans all square roots.

\section{References}

[1] R. Dipper and G. James, 'Representations of Hecke algebras of general linear groups', Proc. London Math. Soc. (3) 52 (1986), 20-52. 
[2] — 'Blocks and idempotents of Hecke algebras of general linear groups', Proc. London Math. Soc. (3) 54 (1987), 57-82.

[3] K. Erdmann and D. K. Nakano, 'Representation type of Hecke algebras of type A', Trans. Amer. Math. Soc. 354 (2002), 275-285 (electronic).

[4] A. Francis, 'The minimal basis for the centre of an Iwahori-Hecke algebra', J. Algebra 221 (1999), $1-28$.

[5] A. Francis and L. Jones, 'On bases of centres of Iwahori-Hecke algebras of the symmetric group', J. Algebra 289 (2005), 42-69.

[6] A. R. Francis and J. J. Graham, 'Centres of Hecke algebras: the Dipper-James conjecture', $J$. Algebra 306 (2006), 244-267.

[7] M. Geck and R. Rouquier, 'Centers and simple modules for Iwahori-Hecke algebras', in: Finite reductive groups (Luminy, 1994) (Birkhäuser Boston, Boston, MA, 1997) pp. 251-272.

[8] L. K. Jones, 'Centers of generic Hecke algebras', Trans. Amer. Math. Soc. 317 (1990), 361-392.

[9] G. E. Murphy, 'The idempotents of the symmetric group and Nakayama's conjecture', J. Algebra 81 (1983), 258-265.

[10] Algebra 152 (1992), 492-513.

[11] _ - 'The representations of Hecke algebras of type $A_{n}$ ', J. Algebra 173 (1995), 97-121.

[12] J. Murray, 'Squares in the centre of the group algebra of a symmetric group', Bull. London Math. Soc. 34 (2002), 155-164.

[13] O. Ogievetsky and P. Pyatov, 'Lecture on Hecke algebras', unpublished, 1999.

[14] A. Ram, 'Seminormal representations of Weyl groups and Iwahori-Hecke algebras', Proc. London Math. Soc. (3) 75 (1997), 99-133.

School of Computing and Mathematics

University of Western Sydney

NSW 1797

Australia

e-mail: a.francis@uws.edu.au
Department of Mathematics

Shippensburg University

Pennsylvania

USA

e-mail: lkjone@ship.edu 